\title{
Respiratory challenge induces high frequency spiking on the static charge sensitive bed (SCSB)
}

\author{
T. Kirjavainen*†, O. Polo*, S. McNamara $*$ K. Vaahtoranta*, C.E. Sullivan
}

Respiratory challenge induces high frequency spiking on the static charge sensitive bed (SCSB). T. Kirjavainen, O. Polo, S. McNamara, K. Vaahtoranta, C.E. Sullivan. CERS Journals Ltd 1996.

ABSTRACT: The static charge sensitive bed (SCSB) is a simple and noninvasive device used for the detection of sleep apnoea. In addition to episodes of apnoea or hypopnoea, heavy snorers commonly present with episodes of high frequency spiking on the SCSB. These spiking episodes have been claimed to represent partial upper airway obstruction during sleep, but the mechanism of their appearance is not known. We studied the SCSB spiking phenomenon in awake subjects during experimental respiratory challenge.

One female and five male volunteers were studied whilst breathing freely, during hypoxia, hypercapnia and inspiratory and expiratory loading. Oxygen saturation, end-tidal carbon dioxide tension, minute ventilation, oesophageal pressure, electrocardiographic activity (ECG), blood pressure and the SCSB signals were monitored.

During free breathing, the SCSB high frequency signal consisted of low amplitude complexes with close time relationship to the cardiac cycle. During respiratory challenge, spiking occurred. These spikes showed no time relationship to the cardiac cycle, but were time-linked to the onset of inspiration or expiration. Spike amplitude correlated with breathing frequency $\left(r^{2}=0.59 ; p<0.005\right)$ and variation in oesophageal pressure $\left(r^{2}=0.57 ; p<0.005\right)$.

We conclude that during quiet, unobstructed breathing the static charge sensitive bed high frequency signal represents cardiac activity (ballistocardiogram), whereas during high-drive breathing high frequency spikes are produced. These spikes are respiratory in origin and are likely to represent fast components of respiratory movements. Our results support the use of static charge sensitive bed spiking as a noninvasive measure of breathing stimulation.

Eur Respir J., 1996, 9, 1810-1815.
Depts of *Physiology and $†$ Medicine, University of Turku, Finland. $\$$ Dept of Medicine, University of Sydney, Australia.

This study was performed at: Centre for Respiratory Failure and Sleep Disorders, Royal Prince Alfred Hospital, Camperdown, Australia.

Correspondence: T. Kirjavainen

Institute of Biomedicine

Dept of Physiology

University of Turku

Kiinamyllynkatu 10

FIN-20520 Turku

Finland

Keywords: Movement

obstructive sleep apnoea

respiration

snoring

static charge sensitive bed

Received: December 81995

Accepted after revision May 111996

SM was supported by the National Health and Medical Research Council of Australia
The static charge sensitive bed (SCSB) is a sensitive movement sensor that is designed for long-term monitoring of gross body, respiratory and heart-related movements [1]. The images of these three different movements are contained within the composite signal generated by the SCSB, and are separated through analogue frequency filtering. The low frequency band (LFB) represents respiratory movements, whereas the high frequency band (HFB) reflects cardiac activity (the ballistocardiogram). In Finland and Sweden, the SCSB has been widely used since 1985 for the assessment of sleep-related breathing disorders $[2,3]$.

Prolonged episodes of SCSB spiking (high amplitude, high frequency deflections) are common in patients with sleep-disordered breathing $[4,5]$, and appear during heavy nonapnoeic or nonhypopnoeic snoring. In a previous study, we evaluated the prevalence of SCSB spiking in 119 consecutive patients who were referred to the Montpellier Sleep and Wake Disorders Unit because of snoring, excessive sleepiness or clinically suspected obstructive sleep apnoea (OSA) [5]. Prolonged episodes of SCSB spiking were observed in $44(53 \%)$ out of 83 patients with OSA and 18 (50\%) out of 36 nonapnoeic snorers. In another study, despite a decrease in the apnoea/hypopnoea index, the frequency of spiking episodes increased after uvulo-palatopharyngoplasty (UPPP) in 10 out of 11 patients with OSA [6]. Conversely, in subjects without nocturnal breathing disorders, SCSB spiking has not been observed [7-11].

Nonapnoeic snorers who present with SCSB spiking have narrower hypopharyngeal airways than controls [12]. Also, the degree of SCSB spiking correlates with intrathoracic pressure variation $\left(r^{2}=0.46\right)$ during obstructed breathing [13]. Therefore, episodes of spiking have been claimed to represent partial upper airway obstruction and have been referred to as the increased respiratory resistance (IRR) pattern [4-6]. However, since the conventional polysomnogram does not differentiate between epochs with and without SCSB spiking [5], this phenomenon is insufficiently described in the literature.

The mechanisms responsible for SCSB spiking are not known. It has been proposed that the appearance of these spikes is a manifestation of increased respiratory variation of the ballistocardiogram [13]. For better characterization 
of the SCSB spiking phenomenon and its pathophysiological significance, we studied the appearance of SCSB spiking during experimental respiratory challenge.

\section{Subjects and methods}

Five males and one female volunteered for the study. Their mean age was 33 years (SD 10, range 23-48 yrs) and mean body mass index (BMI) was $25.2 \mathrm{~kg} \cdot \mathrm{m}^{-2}$ (SD 1.4 , range $23.7-27.4 \mathrm{~kg} \cdot \mathrm{m}^{-2}$ ).

The experiments were performed during the day between 11:00 and 16:00 $\mathrm{h}$, whilst the subjects were awake and without any sleep-deprivation. The subjects were lying supine on a SCSB and were instructed to relax and to avoid body movements. The recording set-up (fig. 1) consisted of continuous monitoring of the SCSB signals, electrocardiogram (ECG), blood pressure, airflow, oesophageal pressure, arterial oxygen saturation $\left(\mathrm{Sa}_{\mathrm{a}} \mathrm{O}_{2}\right)$ and end-tidal carbon dioxide pressure $\left(P \mathrm{ET}, \mathrm{CO}_{2}\right)$. The original signals were digitized, stored and analysed with 12-bit amplitude resolution, $250 \mathrm{samples} \cdot \mathrm{s}^{-1}$ (UniPlot ${ }^{\circledR}$, Unesta, Turku, Finland).

The study protocol was approved by the Ethics Review Committee of the Royal Prince Alfred Hospital, Sydney, Australia. Each subject was studied during seven 5 min periods in the following sequence: 1) a control period was followed by three periods of ventilatory chemostimulation; with 2) hypercapnia; 3) eucapnic hypoxia; and 4) hypercapnic hypoxia. Recordings were also performed during: 5) inspiratory resistive loading; 6) expiratory resistive loading; and 7) combined inspiratory and expiratory loading. Each test period was separated by a $10 \mathrm{~min}$ recovery period. During the control period, the subjects were breathing room air without added resistance for breathing. Hypercapnia was gradually induced until a moderate ventilatory response was achieved, i.e. ventilation above $20 \mathrm{~L} \cdot \mathrm{min}^{-1}$. During the hypercapnic tests, $P$ ET, $\mathrm{CO}_{2}$ ranged 5-10 $\mathrm{mmHg}$ above the level observed during the control period. Hypoxia was also gradually induced until $\mathrm{Sa}_{\mathrm{a}} \mathrm{O}_{2}$ reached $80 \%$ (eucapnic hypoxia) or 85\% (hypercapnic hypoxia). The inspiratory and expiratory loads were increased to a level where the subjects were still able to maintain steady-state breathing over the 5 min period. During respiratory loading, the subjects were advised to keep the inspiratory and expiratory duration similar in order to avoid compensation of resistance by increasing the length of the breathing cycle.

Subjects breathed continuously via a mouthpiece attached to a computer-controlled biased-flow rebreathing circuit. This system was used to produce sustained normoxic hypercapnia, eucapnic hypoxia or hypercapnic hypoxia, and to control $\mathrm{Sa}_{\mathrm{a}} \mathrm{O}_{2}$ and $\mathrm{PET}, \mathrm{CO}_{2}$, and measure ventilation. The principle of the circuit has been described in detail previously [14]. In brief, the breathing circuit included a fixed speed blower motor $\left(50 \mathrm{~L} \cdot \mathrm{min}^{-1}\right)$, a bypassable soda-lime absorber, and a $6 \mathrm{~L}$ flow-through bag connected to a Fleisch No. 3 pneumotachograph with a differential pressure transducer (Validyne ${ }^{\circledR}$ DP-45; Validyne Corp., Northridge, CA, USA). PET, $\mathrm{CO}_{2}$ was measured at the mouthpiece by means of an infrared carbon dioxide analyser (Capnometer ${ }^{\circledR}$ 47210a; Hewlett-Packard, Waltham, MA, USA) and $\mathrm{Sa}_{\mathrm{a}} \mathrm{O}_{2}$ via an ear probe pulse oximeter (Pulse Oximeter ${ }^{\circledR}$; Ohmeda Biox 3700e). A computer monitored the $P$ ET, $\mathrm{CO}_{2}$, and adjusted the degree of $\mathrm{CO}_{2}$ rebreathing accordingly, by controlling the electronic valves that partitioned the air through and past the sodalime absorber. A resistive obstructer connected between the mouthpiece and the biased-flow circuit was used to produce airflow limitation (fig. 1). The obstructer consisted of a valve system with resistances which were separately adjustable for inspiration and expiration.

The static charge sensitive bed (SCSB, Biomatt ${ }^{\circledR}$ ); Biorec, Turku, Finland) consists of a $2 \mathrm{~cm}$ thick movement sensor, which is placed under a normal foam plastic mattress (fig. 1). In the present study, the signal generated by the SCSB was filtered into LFB and HFB, with frequencies ranging $0.25-0.9 \mathrm{~Hz}$ (the peak value \pm 3 $\mathrm{dB})$ and $6-16 \mathrm{~Hz}$, respectively. This filtering is standard for the SCSB method [5]. The response delays of the

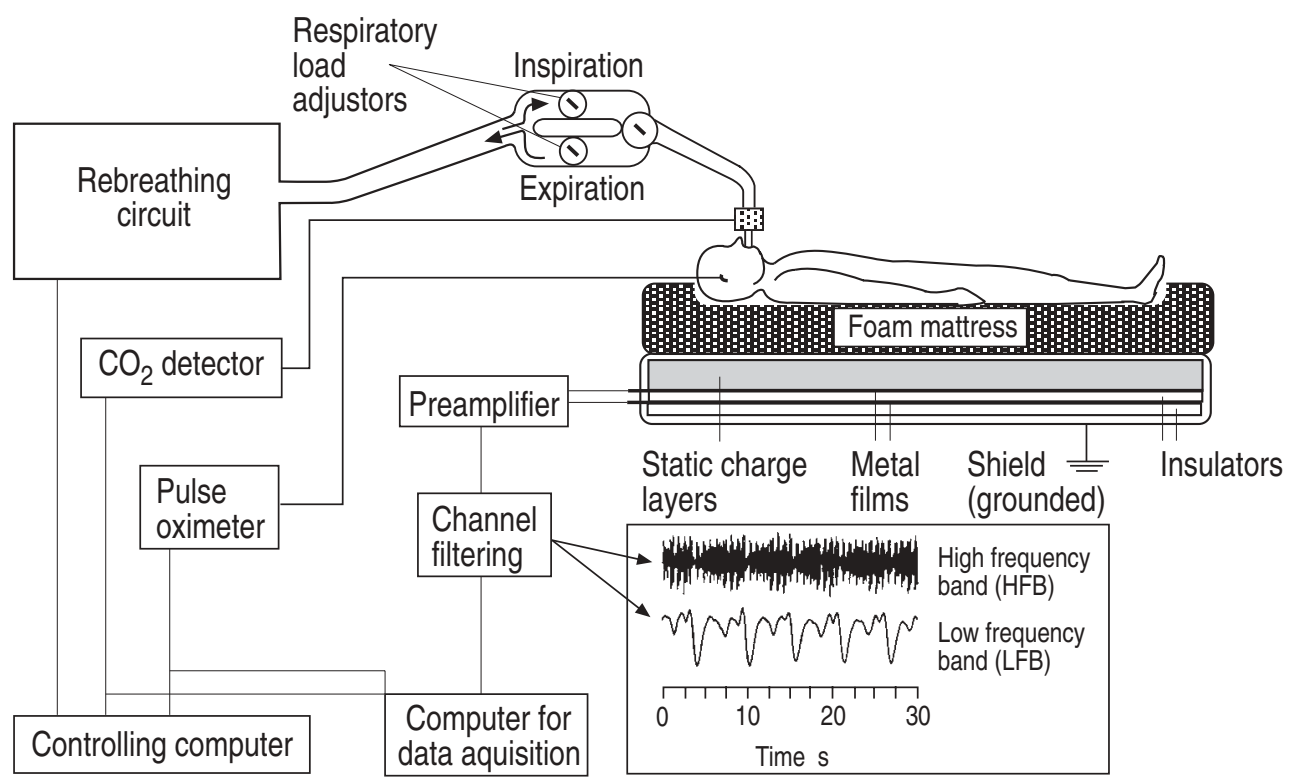

Fig. 1. - The recording set-up with the static charge sensitive bed (SCSB). 
two channels were tested by using $\pm 1 \mathrm{~V}$ square-shaped input signal, which corresponds to the mean input voltage for filters. The latencies of maximal signal amplitudes were $38 \mathrm{~ms}$ for the HFB and $539 \mathrm{~ms}$ for the LFB.

As an estimation of intrapleural pressure, oesophageal pressure was measured using a water-filled, low-pressure, continuous-flow system similar to that used by FLEMALE et al. [15]. The system consisted of a stiff $100 \mathrm{~cm}$ long catheter (Feeding tube FG 8; Indoplas PTY Ltd, Australia), with multiple extra side holes at the tip of the catheter, which was connected to a high pressure $(300 \mathrm{mmHg})$ infusion bag via a pressure transducer (Spectramed P23XL; Statham, USA) and a high resistance tube (Sorenson Intraflo® II; Abbott Critical Care Systems, IL, USA). This system gives a continuous flush flow of $3 \mathrm{~mL} \cdot \mathrm{h}^{-1}$. The catheter was inserted through the nose and fixed to a position where the tip of the catheter was at the distance of 33.5-35 $\mathrm{cm}$ from the nares, approximately $10 \mathrm{~cm}$ above the diaphragm [16]. The pressure transducer was fixed to the level of the mid-axillary line. Calibrations and the linearity of response were tested over the pressure range of $\pm 20 \mathrm{cmH}_{2} \mathrm{O}$ using a water manometer. The system was kept free of air bubbles and mucus by extra flushes with water between the test periods The signal quality during test periods was controlled by observing the cardiac artifacts on the pressure signal.

The blood pressure was measured with a digital cuff (Finapress ${ }^{\circledR}$, Ohmeda 2300; The BOC Group Ins., Louisville, CO, USA) and the ECG was recorded continuously.

\section{Data analysis}

Analysis 1. For the time-lag and spike amplitude analyses, $30 \mathrm{~s}$ of representative recordings, without gross body movements were analysed during the control period and during each test period (fig. 2). The average cardiac frequency, blood pressure, airflow, minute ventilation, breathing frequency, oesophageal pressure variation and the maximal rate of change of the oesophageal pressure during inspiration and expiration of each epoch were determined. Minute ventilation $\left(V^{\prime} \mathrm{E}\right)$ was calculated from the calibrated airflow signal as mean ventilation of inspired and expired air. For the time-lag analyses, the five highest HFB complexes during the control periods and stimulated breathing were selected. The latencies of SCSB spikes were measured from the R-wave of previous ECG QRS-complex (A-latency) and from the previous zero airflow value, which was the onset of either inspiration (Binsp-latency) or expiration (Bexp-latency) The principle of these measurements is presented in figure 3. For the spike amplitude analyses, the reference amplitude for each subject was determined according to the mean of 10 maximal HFB deflection (spike) amplitudes recorded during the control period.

Analysis 2. The SCSB spike amplitudes and the oesophageal pressure variations were also studied during the transition periods from the recovery period to chemostimulated breathing. For this purpose, 10 min periods of increasing respiratory effort were analysed and the maximal SCSB spike amplitude, the integral of HFB signal and the minimum oesophageal pressure were determined every $10 \mathrm{~s}$.

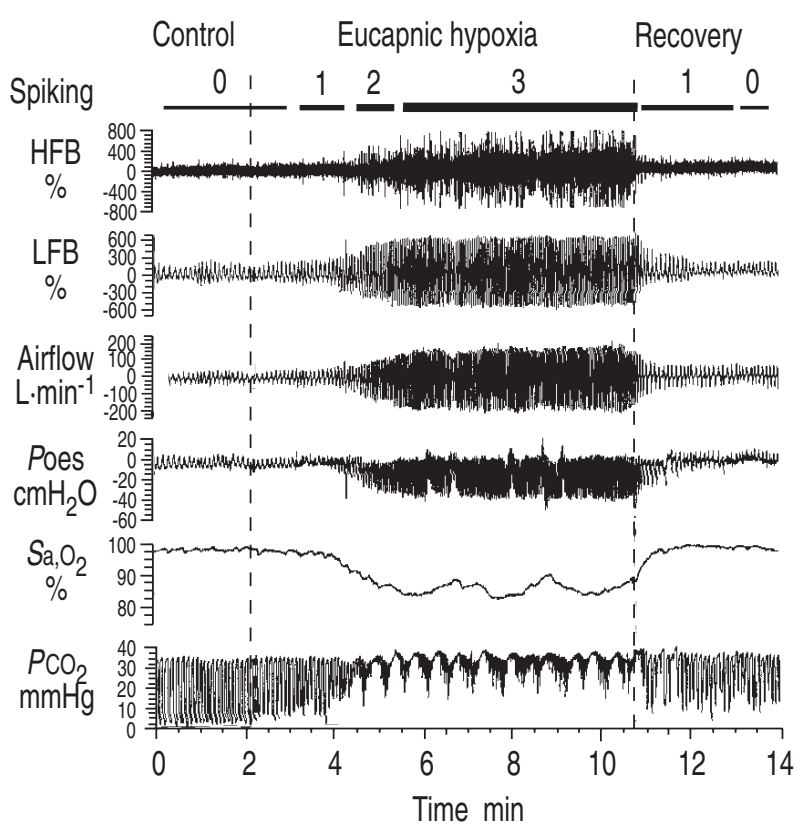

Fig. 2. - An example of a eucapnic hypoxic test period. Gradually increased SCSB spiking disclosed by the high frequency band (HFB) during eucapnic hypoxic stimulation test, together with increasing ventilation and breathing effort. In visual scoring, the SCSB spiking was classified into four categories: $0=$ no spiking; $1=$ light spiking; $2=$ moderate spiking; and 3=heavy spiking. HFB: SCSB high frequency band (\% from control); LFB: SCSB low frequency band (\% from control); $P$ oes: oesophageal pressure; $\mathrm{Sa}_{\mathrm{a}} \mathrm{O}_{2}$ : arterial oxygen saturation; $\mathrm{PCO}_{2}$ : partial pressure of carbon dioxide measured at the mouthpiece. SCSB: static charge sensitive bed.

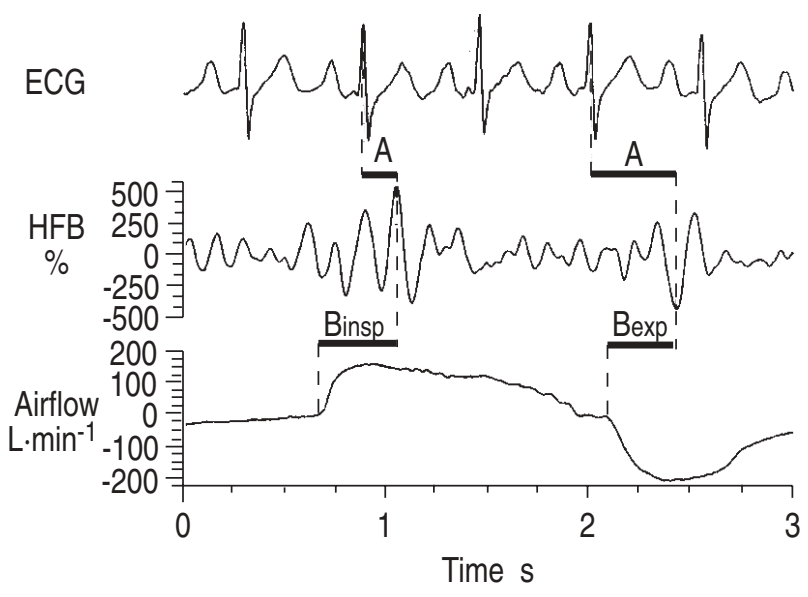

Fig. 3. - The principle of the time-lag analyses: A-latency (A) was measured from the R-wave of the previous ECG QRS-complex to the SCSB spike. B-latency (B) was measured from the onset of previous respiratory effort, which was either inspiration (Binsp) or expiration (Bexp), to the SCSB spike. ECG: electrocardiogram; HFB: SCSB high frequency band (\% from control); SCSB: static charge sensitive bed.

Analysis 3. In clinical sleep studies, the recognition of SCSB spiking is based on visual analysis. Therefore, in the present study the degree of spiking was visually classified into four categories in terms of spike amplitude and frequency: $0=$ no spiking; $1=$ light spiking; $2=$ moderate spiking; and $3=$ heavy spiking (fig. 2). On this scale, the previously used concept of the "increased respiratory resistance" pattern would equal moderate or heavy spiking. The degree of spiking was visually determined from the entire recording, including the control period, the periods of transition as well as the test periods, but 
excluding gross body movements and signal artifacts. Scoring was performed in $30 \mathrm{~s}$ epochs by the same person (TK) blinded to signals other than HFB. The average number of epochs analysed was 78 (SD 5, range 74-87). The degree of SCSB spiking was correlated with the average spike amplitude, breathing frequency and the average minimum oesophageal pressure.

\section{Statistical analyses}

Levene's one-way analysis of variance (ANOVA) was used to compare differences in the variation of $\mathrm{A}$ - and B-latencies. To study the differences between chemostimulated and obstructed breathing and between visual scoring categories of SCSB spiking, the two-factor ANOVA without replication was used. Linear regression analysis by the least square method was used in calculations of correlations between parameters, separately for each subject. The average correlations of subjects are presented. The p-values for the regression were calculated by using Student's t-test. Statistical significance was assumed at p-values of less than 0.05 .

a)

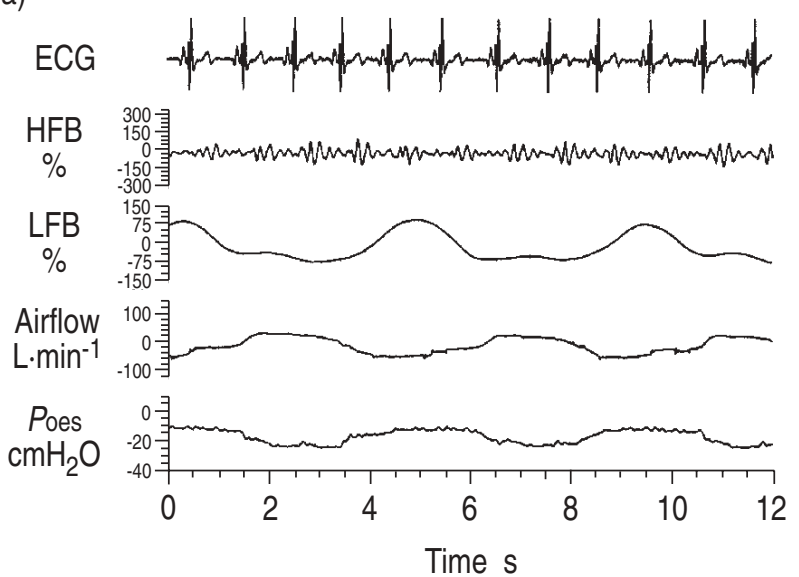

\section{Results}

Appearance of the SCSB spikes in relation to heart and respiratory cycles

Analysis 1. During the control period, breathing was regular and the SCSB high frequency signal showed regular low amplitude complexes closely time-related to ECG (fig. 4a). The mean A-latency (the time delay from the previous ECG R-wave to the low amplitude complexes) of these complexes was $476 \mathrm{~ms}$ (SD 100, range 363-612 ms) (table 1). The appearance of the complexes was unrelated to breathing but their amplitude was modulated by respiration. During inspiration, the amplitude was higher than during expiration (fig. 4a). No spikes were observed during the control period.

Increased respiratory efforts produced by chemostimulation or resistive loading induced typical SCSB spiking, which was similar to that observed during sleep studies in heavy snorers (fig. 2). The spikes appeared synchronously with the onset of either inspiration or expiration, without any time relationship to the ECG (fig. 4b). The variability of A-latencies was higher during chemostimulation or resistive loading than during the control period

b)

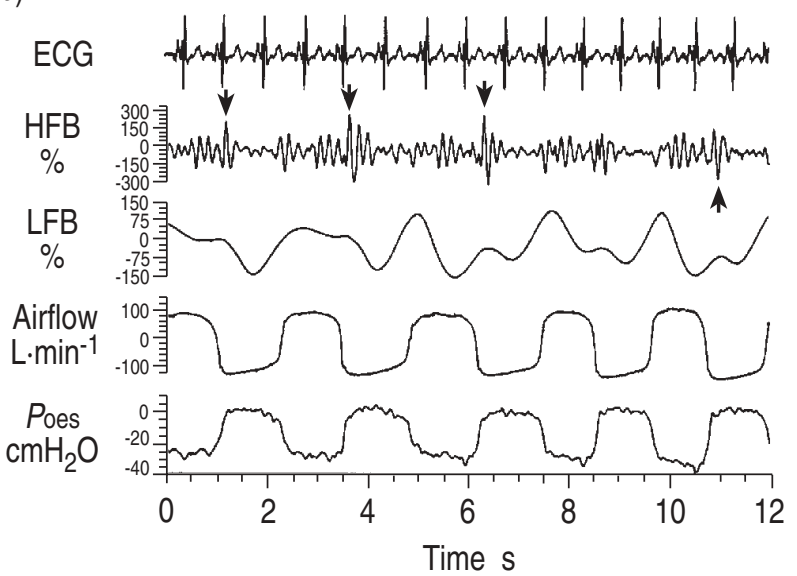

Fig. 4. - A typical recording during: a) control periods; and b) hypercapnic hypoxic periods. During the control period, low-amplitude complexes seen on the HFB were closely time-linked to ECG R-wave. When breathing was stimulated, the SCSB spiking appeared. These spikes showed no time relationship to ECG, but were related to breathing. Spikes are indicated by arrows in the figure. For definitions see legends to figures 1 and 2.

Table 1. - Descriptive average values of each recording period

\begin{tabular}{|c|c|c|c|c|c|c|c|c|c|}
\hline \multirow[b]{2}{*}{ Period } & \multicolumn{3}{|c|}{ Heart } & \multicolumn{5}{|c|}{ Respiration } & \multirow{2}{*}{$\begin{array}{c}\text { HFB } \\
\begin{array}{c}\text { Spike ampl } \\
\%\end{array}\end{array}$} \\
\hline & $\begin{array}{c}f \mathrm{C} \\
\text { beats.min }\end{array}$ & $\begin{array}{l}\text { A-Lat } \\
\text { ms }\end{array}$ & $\begin{array}{c}\mathrm{A}-\mathrm{Var} \\
10^{-3}\end{array}$ & $\begin{array}{c}f \mathrm{R} \\
\text { breaths.min-1 }\end{array}$ & $\begin{array}{l}\text { B-Lat } \\
1 \mathrm{~ms}\end{array}$ & $\begin{array}{c}\mathrm{B}-\mathrm{Var} \\
10^{-3}\end{array}$ & $\begin{array}{c}V^{\prime} \mathrm{E} \\
\mathrm{L} \cdot \mathrm{min}^{-1}\end{array}$ & $\begin{array}{c}\Delta P_{\text {oes }} \\
\mathrm{cmH}_{2} \mathrm{O}\end{array}$ & \\
\hline Control & 70 & 476 & 3 & 14 & 1197 & 1060 & 9 & 7 & 106 \\
\hline Hypercapnia & 72 & 463 & 32 & 19 & 571 & 476 & 32 & 22 & 200 \\
\hline Hypoxia & 81 & 439 & 25 & 20 & 704 & 498 & 27 & 18 & 194 \\
\hline Hypercapnic hypoxia & 81 & 367 & 28 & 23 & 528 & 379 & 43 & 28 & 343 \\
\hline Inspiratory load & 77 & 421 & 56 & 21 & 401 & 185 & 17 & 32 & 225 \\
\hline Expiratory load & 72 & 343 & 46 & 21 & 126 & 15 & 17 & 27 & 216 \\
\hline Inspiratory and expiratory loads & 78 & 363 & 46 & 25 & 202 & 19 & 18 & 43 & 281 \\
\hline
\end{tabular}

$f_{C}$ : cardiac frequency; A-Lat: time latency of a SCSB spike from the previous ECG R-wave; A-Var: mean variance of A-latency; B-Lat: time latency of a SCSB spike from the beginning of previous respiratory effort; B-Var: mean variance of B-latency; $V^{\prime} \mathrm{E}$ : minute ventilation; $\Delta P_{\text {oes: }}$ oesophageal pressure difference between inspiration and expiration; $f \mathrm{R}$ : breathing frequency; Spike ampl: mean amplitude of SCSB spikes during the epoch; SCSB: static charge sensitive bed; ECG: electrocardiogram. 
$(\mathrm{p}<0.0001)$ (table 1). The lack of time relationship between the SCSB spiking and the ECG R-wave during stimulated breathing suggests that the SCSB spikes are not of cardiac origin.

The B-latencies (the time delay from the onset of inspiration or expiration to the SCSB spike) decreased as the spike amplitude increased during chemostimulation or resistive loading. The variability of B-latencies was smaller both during chemostimulation periods $(\mathrm{p}=0.01)$ and resistive loading $(\mathrm{p}<0.0001)$ than during the control period (table 1). This implies that SCSB spikes were more dependent on respiration during the respiratory challenge periods than the low amplitude complexes observed during the control period.

SCSB spikes appeared both in inspiration and expiration during all challenge periods, but their number was greater during expiration, except during simultaneous inspiratory and expiratory loading. The type of respiratory stimulation did not have significant influence on the distribution of the SCSB spikes.

\section{Determinants of the SCSB spike amplitude}

Analysis 1. The correlations between spike amplitude and the parameters measured are presented in table 2 . The best correlations were observed with breathing frequency $\left(\mathrm{r}^{2}=0.59 ; \mathrm{p}<0.005\right)$ and with the average oesophageal pressure difference during the epoch $\left(\mathrm{r}^{2}=0.57 ; \mathrm{p}<\right.$ $0.005)$. The correlations of spike amplitude to airflow and minute ventilation (and thus to the thoracic movement) were only weak, especially during resistive loading.

Table 2. - Correlation of SCSB spike amplitude with the recorded respiratory and cardiovascular parameters during control and test periods

\begin{tabular}{lccc}
\hline & \multicolumn{2}{c}{ Correlation with spike amplitude } \\
\cline { 2 - 3 } & \multicolumn{2}{c}{$\mathrm{r}^{2}$} & \\
\cline { 2 - 3 } & mean $\pm \mathrm{SD}$ & range & p-value \\
\hline Breathing frequency & $0.59 \pm 0.27$ & $0.20-0.84$ & $<0.005$ \\
$\Delta P_{\text {oes }}$ & $0.57 \pm 0.25$ & $0.19-0.93$ & $<0.005$ \\
Expiratory slope of $P_{\text {oes }}$ & $0.55 \pm 0.20$ & $0.32-0.79$ & $<0.005$ \\
Inspiratory slope of $P$ oes & $0.54 \pm 0.17$ & $0.24-0.71$ & $<0.001$ \\
Minute ventilation & $0.37 \pm 0.31$ & $0.00-0.70$ & $<0.05$ \\
$\Delta$ Blood pressure & $0.15 \pm 0.11$ & $0.01-0.32$ & $<0.05$ \\
Airflow at the spike & $0.10 \pm 0.13$ & $0.00-0.29$ & NS \\
\hline
\end{tabular}

$\Delta$ Blood pressure: difference between pre- and post-spike systolic blood pressure; Ns: nonsignificant. For further definitions see legend to table 1 .
Analysis 2. The correlations $\left(\mathrm{r}^{2}\right)$ between SCSB spike amplitude and oesophageal pressure within the periods of transition from recovery to chemostimulation were: 0.48 (SD 0.27, range $0.22-0.91 ; \mathrm{p}<0.01$ ) during hypercapnia; 0.45 (sD 0.31 , range $0.15-0.92 ; \mathrm{p}<0.02$ ) during eucapnic hypoxia; and 0.65 (SD 0.11, range $0.46-0.77$; $\mathrm{p}<0.001$ ) during hypercapnic hypoxia. The higher the level of breathing stimulation (indicated by a more negative inspiratory oesophageal pressure) the better was the correlation $\left(r^{2}=0.51 ; p<0.0001\right)$. The integral of HFB signal correlated more strongly with oesophageal pressure than the SCSB spike amplitude $(\mathrm{p}<0.0001)$, and the respective $\mathrm{r}^{2}$ values were: 0.59 (SD 0.23, range $0.33-0.91 ; \mathrm{p}<0.002$ ) during hypercapnia; 0.54 (SD 0.29, range 0.14-0.88; $\mathrm{p}<$ 0.01 ) during eucapnic hypoxia; and 0.76 (SD 0.12 , range $0.55-0.92 ; \mathrm{p}<0.001)$ during hypercapnic hypoxia.

\section{Visual analysis of the SCSB spiking}

Analysis 3. The average correlation $\left(\mathrm{r}^{2}\right)$ between visually scored degree of SCSB spiking and breathing frequency was 0.87 (SD 0.11, range 0.66-0.95; $\mathrm{p}<0.0001$ ) and 0.83 (sD 0.19, range 0.46-0.97; $\mathrm{p}<0.001$ ) between spiking and average minimum oesophageal pressure. The breathing frequencies $(\mathrm{p}<0.0001)$ and the minimum oesophageal pressures $(p<0.0001)$ were significantly different in the four categories of spiking, and high levels of oesophageal pressure variations were needed to produce moderate or heavy spiking (table 3).

\section{Discussion}

In heavy snorers, the static charge sensitive bed (SCSB) records episodes of breathing abnormalities, that cannot be defined by existing concepts of apnoea or hypopnoea. These episodes are characterized by increasing appearance of high amplitude high frequency movements (SCSB spikes) [5]. In the present study, the mechanisms of appearance of these spikes and the determinants of their amplitude were evaluated. The SCSB spikes were found to be of respiratory origin, and induced during strong respiratory efforts, irrespective of the type of respiratory stimulus. The degree of spiking correlated consistently with the degree of respiratory effort measured by the oesophageal pressure variation.

During control breathing, no SCSB spikes were observed. This is consistent with the findings of the SCSB sleep recordings in controls [7-11]. High frequency movements, detected by the SCSB as low amplitude complexes

Table 3. - Average SCSB spike amplitude, respiratory frequency and minimum oesophageal pressure during epochs of different degree of SCSB spiking

\begin{tabular}{|c|c|c|c|c|c|c|c|c|c|c|c|c|}
\hline \multirow{2}{*}{$\begin{array}{l}\text { Subject } \\
\text { No. }\end{array}$} & \multicolumn{4}{|c|}{ SCSB spike amplitude \% } & \multicolumn{4}{|c|}{$f_{\mathrm{R}}$ breaths $\cdot \mathrm{min}^{-1}$} & \multicolumn{4}{|c|}{ Minimum $P_{\text {oes }} \quad \mathrm{cmH}_{2} \mathrm{O}$} \\
\hline & 0 & 1 & 2 & 3 & 0 & 1 & 2 & 3 & 0 & 1 & 2 & 3 \\
\hline 1 & 118 & 179 & 356 & 541 & 14 & 17 & 22 & 24 & -16 & -20 & -23 & -26 \\
\hline 2 & 122 & 167 & 249 & 476 & 15 & 19 & 24 & 27 & -15 & -23 & -32 & -44 \\
\hline 3 & 114 & 149 & 214 & 392 & 16 & 14 & 15 & 23 & -14 & -20 & -25 & -48 \\
\hline 4 & 92 & 122 & 213 & 314 & 12 & 15 & 18 & 17 & -9 & -15 & -23 & -28 \\
\hline 5 & 138 & 204 & 343 & 693 & 10 & 13 & 16 & 24 & -12 & -20 & -30 & -43 \\
\hline 6 & 108 & 137 & 184 & 263 & 14 & 16 & 18 & 18 & -9 & -13 & -22 & -30 \\
\hline Mean & 115 & 160 & 260 & 447 & 13 & 15 & 19 & 22 & -12 & -19 & -26 & -36 \\
\hline
\end{tabular}

Categories of SCSB spiking: $0=$ none; $1=$ light; $2=$ moderate; $3=$ heavy. SCSB: static charge sensitive bed; $f \mathrm{R}$ : respiratory frequency; Poes: oesophageal pressure. 
during control breathing, were closely time-linked to the ECG R-wave and are, therefore, probably of cardiac origin. These movements arise from heart contraction and displacement of blood in the great vessels [17, 18].

The appearance of the SCSB spiking during respiratory challenge was dependent on the degree of respiratory response rather than the type of respiratory stimulus. In contrast to the low amplitude complexes observed during control breathing, these spikes were not time-related to the ECG R-wave, but emerged with a short latency after onset of inspiration or expiration (fig. 4). Thus, the spikes represent fast components of respiratory movements, which seem to be produced only when there is high respiratory drive. The present study did not indicate which specific movements of the rib cage, abdomen or diaphragm would be responsible for the fast components observed. With high breathing frequencies, it is understandable that the thoracic movements are fast enough to produce spiking. However, since respirationrelated spikes were also produced during low frequency high effort breathing, changes in the onset pattern of inspiration or expiration are also likely to be involved.

Once the SCSB spikes appeared, their amplitude showed marked dynamics, which were highly correlated with breathing frequency and variation in oesophageal pressure. The gradual increase of the spike amplitude was evident during the transition periods, where there was also a gradually increasing ventilatory response to hypercapnia or hypoxia (fig. 2). This implies that the SCSB spike amplitude could be used as an estimate of the degree of breathing stimulation. The visual scoring system used in the clinical sleep studies was evaluated by determining the average levels of oesophageal pressure variation and breathing frequencies that corresponded to the four categories of spiking (table 3 ). The visual score of the SCSB spiking and the integral of the high frequency signal were better than the calculated spike amplitudes to estimate the oesophageal pressure variation. These pressure levels were obtained in awake, normal volunteers lying supine and are, therefore, not directly applicable to obese snorers during sleep. However, the present findings suggest that the previously defined IRR pattern used in clinical sleep studies to describe moderate or heavy SCSB spiking $[4-6,12]$ is associated with a marked degree of respiratory stimulation. Although the IRR pattern is similarly observed when lying supine or on the side [5], the effect of body position on the sensitivity of the spiking as an indicator of respiratory stimulation remains to be confirmed.

In the present study, we have shown that SCSB spiking correlates well with the most direct measure of respiratory effort, which is the variation in oesophageal pressure [19]. There are few alternatives to the measurement of oesophageal pressure, which is often considered too invasive for routine sleep studies. Surface electromyograms (EMG) of the diaphragm or intercostal muscles produce high quality information about the respiratory effort at their best but problems are encountered, particularly in obese subjects [19]. The static charge sensitive bed is a noninvasive method and provides a robust signal for long-term monitoring of respiration. In addition to disclosing episodes of apnoea or hypopnoea during sleep, the present study suggests that the high frequency band of the static charge sensitive bed contains pertinent information that can be employed to assess the degree of respiratory drive.

\section{References}

1. Alihanka J, Vaahtoranta K, Saarikivi I. A new method for long-term monitoring of the ballistocardiogram, heart rate, and respiration. Am J Physiol 1981; 240 (5): 384-392.

2. Polo O, Brissaud L, Sales B, Besset A, Billiard M. The validity of the static charge sensitive bed in detecting obstructive sleep apnoeas. Eur Respir J 1988; 1: 330-336.

3. Svanborg E, Larsson H, Carlsson-Nordlander B, Pirskanen R. A limited diagnostic investigation for obstructive sleep apnea syndrome: oximeter and static charge sensitive bed. Chest 1990; 98: 1341-1345.

4. Alihanka J. Basic principles for analyzing and scoring Bio-Matt (SCSB) recordings. Ann Universitatis Turkuensis 1987; D26: 1-129.

5. Polo O. Partial upper airway obstruction during sleep: studies with the static charge sensitive bed (SCSB). Acta Physiol Scand 1992; 606 (Suppl.): 1-118

6. Polo O, Brissaud L, Fraga J, Déjean Y, Billiard M. Partial upper airway obstruction in sleep after uvulopalatopharyngoplasty. Arch Otolaryngol Head Neck Surg 1989; 115: 1350-1354.

7. Hyyppä MT, Kronholm E. Sleep movements and poor sleep in patients with nonspecific somatic complaints. II. Affective disorders and sleep quality. J Psychosom Res 1987; 31: 631-637.

8. Lauerma H, Lehtinen I, Lehtinen $\mathrm{P}$, et al. Laterality of motor activity during normal and disturbed sleep. Biol Psychiatry 1992; 32: 191-194.

9. Sjöholm T, Polo O, Alihanka J. Sleep movements in teethgrinders. J Craniomandib Disord Facial Oral Pain 1992; 6: 184-191.

10. Lauerma H, Kaartinen J, Polo O, Sallinen M, Lyytinen H. Asymmetry of instructed motor response to auditory stimuli during sleep. Sleep 1994; 17 (5): 444-448.

11. Sjöholm T, Lehtinen I, Helenius H. Masseter muscle activity in diagnosed sleep bruxists compared with nonsymptomatic controls. J Sleep Res 1995; 4: 48-55.

12. Polo O, Tafti M, Fraga J, Porkka KVK, Déjean Y, Billiard M. Why don't all heavy snorers have obstructive sleep apnea? Am Rev Respir Dis 1991; 143: 1288-1293.

13. Polo O, Tafti M, Hämäläinen M, Vaahtoranta K, Alihanka J. Respiratory variation of the ballistocardiogram during increased respiratory load and voluntary central apnoea. Eur Respir J 1992; 5: 257-262.

14. Berthon-Jones M, Sullivan CE. Ventilation and arousal responses to hypercapnia in normal sleeping humans. $J$ Appl Physiol 1984, 57: 59-67.

15. Flemale A, Gillard C, Dierckx JP. Comparison of central venous, oesophageal and mouth occlusion pressures with water-filled catheters for estimating pleural pressure changes in healthy adults. Eur Respir J 1988; 1 (1): 51-57.

16. Irvin CG, Sampson M, Engel L, Grassino AE. Effect of breathing pattern on esophageal pressure gradients in humans. J Appl Physiol: Respirat Environ Exercise Physiol 1984; 57 (1): 168-175.

17. Heald CB, Tucker WS. The recoil curves as shown by the hot wire microphone. Proc Roy Soc Med 1922; 93: 281.

18. Starr I, Friedland CK. On cause of respiratory variation of ballistocardiogram with note on sinus arrhythmia. $J$ Clin Invest 1946; 25: 53-64.

19. Stradling JR. Sleep studies for sleep-related breathing disorders. J Sleep Res 1992; 1: 265-273. 